\title{
LES MÉTHODES DE MESURES DE RÉSISTIVITÉS VOLUMIQUES ET SUPERFICIELLES EN COURANT ALTERNATIF
}

\author{
F. EHRBURGER-DOLLE
}

Centre de Recherches sur la Physico-Chimie des Surfaces Solides

CNRS, 24, avenue du Président-Kennedy, 68-Mulhouse

(Reçu le 30 septembre 1971, révisé le 12 janvier 1972)

\begin{abstract}
Résumé. - Dans cette étude bibliographique, l'auteur s'est proposé d'examiner les différentes méthodes de mesure de résistivité en courant alternatif. On a ainsi passé en revue les mesures par courants de Foucault et les mesures en hyperfréquences. Les premières sont généralement utilisées pour étudier la résistivité volumique d'un échantillon et présentent l'avantage sur les mesures en courant continu de ne nécessiter aucun contact électrique ; au contraire, en hyperfréquence, seule la résistivité superficielle peut être obtenue.
\end{abstract}

\begin{abstract}
In this bibliography review, the author has examined the different methods available to resistivity measurements with alternative current. Both the eddy-current methods and the UHF methods are reviewed. The former are generally used to study the bulk resistivity with the advantage of a direct current requiring no electrical contact. The UHF methods on the other hand, permit one to obtain only the surface resistivity.
\end{abstract}

Introduction. - Nous avons entrepris ce travail bibliographique dans un double but: d'une part, pour faciliter le choix d'une méthode de mesure de conductivité de surface d'un échantillon conducteur massique ou à l'état divisé, d'autre part, pour essayer d'en déduire une méthode de mesure de conductivité de poudre faiblement conductrice. Dans ce deuxième cas plus particulièrement, l'intérêt d'une mesure par courants induits réside dans le fait qu'elle ne nécessite aucun contact électrique qui fausse souvent l'interprétation des résultats.

Les courants de haute fréquence induits dans des échantillons bons conducteurs sont confinés au voisinage de la surface par suite de l'effet de peau de sorte que les propriétés électriques déduites de ces mesures sont spécifiques de la zone superficielle et peuvent varier par suite de différentes modifications physiques ou chimiques de la surface (influence du polissage, oxydation, adsorption de gaz, irradiation, etc...).

Cette étude est essentiellement constituée de deux parties : dans une première partie, nous analyserons les différentes méthodes de mesure de résistivité par courants de Foucault et dans une deuxième partie, nous envisagerons l'application possible des hyperfréquences à l'étude des propriétés électriques superficielles.

1. Rappels théoriques. - 1.1 EQUATIONS GÉNÉRALES. - La propagation des ondes électromagnétiques (caractérisées par l'ensemble des 4 vecteurs E, D, B, H) dans le vide ou dans un milieu de perméabilité $\mu$, de conductivité $\sigma$, de permittivité $\varepsilon$, est régie par les équations de Maxwell [1]

$$
\begin{gathered}
\operatorname{rot} \mathbf{E}=-\frac{\partial \mathbf{B}}{\partial t} \\
\operatorname{rot} \mathbf{H}=\mathbf{J}+\frac{\partial \mathbf{D}}{\partial t} \\
\operatorname{div} \mathbf{D}=\rho \\
\operatorname{div} \mathbf{B}=0
\end{gathered}
$$

et, dans un milieu isotrope :

$$
\begin{aligned}
& \mathbf{D}=\varepsilon \mathbf{E} \\
& \mathbf{B}=\mu \mathbf{H}
\end{aligned}
$$

la loi d'Ohm s'écrit :

$$
\mathbf{J}=\sigma \mathbf{E}
$$

et en l'absence de sources $(\rho=0): \operatorname{div} \mathbf{D}=0$.

Ces 7 équations peuvent se contracter en :

$$
\begin{gathered}
\operatorname{rot} \mathbf{E}=-\mu \frac{\partial \mathbf{H}}{\partial t} \\
\operatorname{rot} \mathbf{H}=\sigma \mathbf{E}+\varepsilon \frac{\partial \mathbf{E}}{\partial t} \\
\operatorname{div} \varepsilon \mathbf{E}=0 \\
\operatorname{div} \mu \mathbf{H}=0 .
\end{gathered}
$$

Dans le cas particulier d'un champ électromagnétique fonction périodique du temps, les champs élec- 
triques et magnétiques peuvent se mettre sous la forme suivante :

$$
\begin{aligned}
& \mathbf{E}(x, y, z, t)=\boldsymbol{E}(x, y, z) \mathrm{e}^{j \omega t} \\
& \mathbf{H}(x, y, z, t)=\boldsymbol{J}(x, y, z) \mathrm{e}^{j \omega t} .
\end{aligned}
$$

Les opérateurs de dérivation par rapport au temps sont alors remplacés par $j \omega$ et les équations de Mawxell s'écrivent :

$$
\begin{aligned}
\operatorname{rot} \boldsymbol{E} & =-\mathrm{j} \omega \mu \mathfrak{H} \\
\operatorname{rot} \mathscr{H} & =(\sigma+j \omega \varepsilon) \boldsymbol{E} \\
\operatorname{div} \mu \mathfrak{H} & =0 \\
\operatorname{div} \varepsilon \boldsymbol{E} & =0
\end{aligned}
$$

ou sous la forme générale de l'équation de propagation des ondes :

$$
\begin{aligned}
\Delta \mathcal{E}-\gamma^{2} \boldsymbol{E} & =0 \\
\Delta \mathscr{H}-\gamma^{2} \mathfrak{H} & =0
\end{aligned}
$$

avec :

$$
\gamma=\sqrt{-\omega^{2} \mu \varepsilon+j \omega \mu \sigma}=\alpha+j \beta .
$$

La valeur de $\gamma$ peut être obtenue dans les cas particuliers :

- isolant parfait

$$
(\sigma=0) \quad \alpha=0 \quad \gamma=j \beta=\sqrt{-\omega^{2} \mu \varepsilon}
$$

- conducteur parfait

$$
\gamma=\sqrt{j \omega \mu \sigma}=(1+j) \sqrt{\frac{\omega \mu \sigma}{2}}
$$

c'est-à-dire $|\alpha|=|\beta|=\sqrt{\pi \mu \sigma f}$ (où $f$ est la fréquence).

1.2 DÉFINITION DE LA PROFONDEUR DE PÉNÉTRATION. - Si on choisit l'axe $\mathrm{Oz}$ comme axe de propagation de l'onde, les champs électriques et magnétiques varient en $\exp (j \omega t)-\gamma z)$. L'atténuation de l'onde est donnée par le facteur $\mathrm{e}^{-\alpha z}$ et par définition, la profondeur de pénétration nominale $\delta$ est la valeur de $z$ pour laquelle les champs électrique et magnétique sont atténués dans un rapport $1 / e$ : d'où

$$
\delta=\frac{1}{\alpha}
$$

Dans le cas d'un conducteur parfait $(\sigma \gg \varepsilon \omega)$ la profondeur de pénétration est donnée par la relation suivante :

$$
\delta=\frac{1}{\sqrt{\pi \mu \sigma f}} .
$$

Par conséquent, en haute fréquence, seule une faible épaisseur d'un matériau conducteur est parcourue par le courant. Dans le cuivre par exemple, la profondeur de pénétration est de $60 \mu$ à $1 \mathrm{MHz}$ et de $0,6 \mu$ à $10 \mathrm{GHz}$; elle dépend également de la température par l'intermédiaire de $\sigma$. Cependant l'action combinée des variables de fréquence et température n'entraîne pas une diminution indéfinie de la profondeur de pénétration.

Lorsqu'elle devient égale ou inférieure au libre parcours moyen des électrons du métal, la loi d'Ohm $J=\sigma E$ n'est plus valable, $\delta$ diminue beaucoup plus lentement. Ceci constitue l'effet de peau anormal étudié principalement par London [2], Pippard [3] ; une théorie plus complète a été faite par Reuter et Sondheimer [4].

1.2 DÉFINITION DE L'IMPÉDANCE DE SURFACE D'UN MÉTAL. - Par suite de la conductivité finie du métal, les courants induits par le champ magnétique existant à la surface créent un faible champ électrique. L'impédance de surface représentera, par définition, le rapport entre l'intensité de ce champ électrique et celle du champ magnétique. En supposant, comme précédemment, la surface métallique placée dans un plan xOy et en admettant que les champs et les courants disparaissent quand $z$ tend vers l'infini, l'application du théorème de Stokes le long d'un contour parallèle à $H$, entre les valeurs $z=0$ et $z$ infini, conduit à l'expression

$$
\int J \mathrm{~d} z=H_{0}
$$

où $H_{0}$ est le champ magnétique à la surface. Dans cette expression, on a négligé le courant de déplacement. L'impédance de surface $Z=R_{\mathrm{s}}+j X_{\mathrm{s}}$, est alors d'après la définition précédente :

$$
Z=\frac{E_{0}}{\int J \mathrm{~d} z}=\frac{E_{0}}{H_{0}} .
$$

L'expression de $Z$ est obtenue en calculant $E_{0}$ et $H_{0}$. Ainsi la résistance de surface est donnée par l'expression :

$$
R_{\mathrm{s}}=\frac{1}{\sigma \delta}
$$

2. Mesures de conductivité par courants de Foucault. - 2.1 PRINCIPE DES DIFFÉRENTES MÉTHODES. Un échantillon conducteur, soumis à un champ électromagnétique variable, est le siège de courants induits tendant à s'opposer à la variation de flux. Cet effet, lié directement à la résistivité de l'échantillon, peut être mesuré de différentes façons. Nous distinguerons essentiellement deux catégories de mesures :

- les mesures faites en régime permanent, consistant à mesurer l'auto-inductance d'une bobine ou à mesurer l'inductance mutuelle de deux bobines entourant l'échantillon, méthodes que nous appellerons, dans la suite de l'exposé, méthodes statiques.

- les mesures faites en régime transitoire, que nous qualifierons de méthodes dynamiques, consistant à mesurer soit l'amortissement des courants de Foucault dans l'échantillon après une brusque variation $\mathrm{du}$ champ magnétique extérieur, soit l'amortissement 
du mouvement de l'échantillon dans un champ magnétique constant.

2.2 MÉTHOdes STATIQUeS (RÉFÉRENCES [5] A [15]). Rorschach et Herlin [5] d'une part, van der Berg et van den Marel [6] d'autre part, semblent avoir été les premiers à faire des mesures de résistivité en étudiant la variation de l'inductance mutuelle de deux bobines. Ces derniers [6], pour réaliser l'équilibre du pont de mesure, ont compensé la perte de flux dans les bobines coaxiales, dues à la présence de l'échantillon, par le changement du nombre de spires d'une des bobines, le nombre de tours étant proportionnel à $\sqrt{\rho / f}$, où $\rho$ est la résistivité et $f$ la fréquence.

Ces auteurs insistent cependant sur le fait que la valeur de $\rho$ ainsi déterminée est, par suite du confinement des courants au voisinage de la surface de l'échantillon, généralement supérieure à la résistivité massique, à cause des défauts ou des contaminants superficiels.

Zimmerman [7] par contre a mesuré la variation de l'inductance et de la résistance d'une bobine entourant un échantillon cylindrique de cuivre et d'un alliage $\mathrm{Cu}-\mathrm{Mn}$, par un pont de mesure. La tension alternative est délivrée par un oscillateur de fréquence variant entre $7 \mathrm{~Hz}$ et $200 \mathrm{~Hz}$. L'ensemble bobine-échantillon est placé dans l'hélium liquide. Les auteurs ont tracé la courbe de variation de la résistivité entre $4,2^{\circ} \mathrm{K}$ et $1{ }^{\circ} \mathrm{K}$. La valeur de la résistivité à partir des mesures de l'inductance et de la résistance est obtenue de la façon suivante :

Soient $L_{0}$ et $R_{0}, L$ et $R$ les inductances et résistances de la bobine seule et de l'ensemble bobine-échantillon respectivement; on peut écrire

$$
L=L_{0}+L^{\prime} \quad \text { et } \quad R=R_{0}+R^{\prime} \text {. }
$$

Les contributions $L^{\prime}$ et $R^{\prime}$ dues à la présence de l'échantillon peuvent être calculées pour des formes simples, cylindriques ou sphériques.

- Echantillon cylindrique :

$$
\begin{aligned}
& K \frac{R^{\prime}}{\omega L_{0}}=\frac{2}{x} \frac{M_{1}(x)}{M_{0}(x)} \sin \left[\theta_{1}(x)-\theta_{0}(x)-\frac{3 \pi}{4}\right] \\
& K \frac{L^{\prime}}{\omega L_{0}}=\frac{2}{x} \frac{M_{1}(x)}{M_{0}(x)} \cos \left[\theta_{1}(x)-\theta_{0}(x)-\frac{3 \pi}{4}\right]-1 .
\end{aligned}
$$

- Echantillon sphérique ou sphéroïdal (axe parallèle au champ)

$$
\begin{aligned}
& K \frac{R^{\prime}}{\omega L_{0}}=\frac{6}{y^{2}}\left[1-\frac{y}{2} \frac{\operatorname{sh} y+\sin y}{2 \operatorname{ch} y-\cos y}\right] \\
& K \frac{L^{\prime}}{\omega L_{0}}=\frac{3 \operatorname{sh} y-\sin y}{y \operatorname{ch} y-\cos y}-1 .
\end{aligned}
$$

Dans ces expressions :

$$
\begin{aligned}
& y^{2}=2 x^{2}=2 \omega \sigma \mu_{0} a^{2} \\
& a \quad \text { rayon de l'échantillon } \\
& \sigma \quad \text { conductivité } \\
& \mu_{0} \quad 4 \pi 10^{-7} \mathrm{H} / \mathrm{m} .
\end{aligned}
$$

$M_{0}(x), \theta_{0}(x), M_{1}(x), \theta_{1}(x)$ sont les composantes polaires des fonctions de Bessel $J_{0}\left(j^{3 / 2} x\right)$ et $J_{1}\left(j^{3 / 2} x\right)$.

$K$ est un facteur assez difficile à évaluer qui, dans le cas du cylindre infini, est approximativement égal au rapport des sections droites de la bobine et de l'échantillon et qui, dans le cas de la sphère peut être défini par la quantité $A^{2} / 2 a^{3}$, si on remplace dans les relations (25) et (26) $L_{0}$ par la valeur de l'inductance par unité de longueur ( $A$ étant le rayon de la bobine).

Une expérience semblable est décrite dans l'article de E. W. et H. H. Johnson [8] dont le but est cependant différent : il s'agit de mesurer la variation de la résistivité en fonction de la distance à la surface. L'échantillon cylindrique de cuivre est placé dans une bobine (Fig. 1), dont la variation d'inductance est mesurée

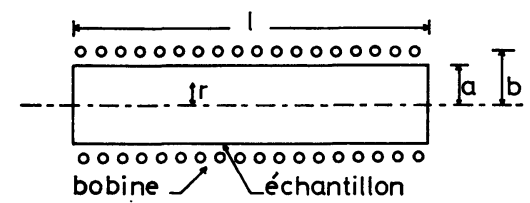

FIG. 1. - Coupe montrant la disposition de l'échantillon dans la bobine dans le cas de l'expérience décrite par Johnson [8].

dans un pont de Owen [9]. A température ambiante, la profondeur de pénétration aux fréquences considérées $(100 \mathrm{~Hz}$ à $100 \mathrm{kHz})$ est encore importante, c'est pourquoi les mesures sont faites à la température de l'azote liquide. Le flux magnétique $\varphi$ à l'intérieur de l'échantillon est déterminé par l'équation différentielle suivante :

$$
\frac{\mathrm{d}^{2} \varphi}{\mathrm{d} x^{2}}-\frac{1}{x} \frac{\mathrm{d} \varphi}{\mathrm{d} x}-j \frac{m^{2} a^{2}}{g(x)} \varphi=0
$$

dans cette expression :

$x=r / a$, variable réduite caractérisant la position d'un point de l'échantillon par rapport à l'axe du cylindre de rayon $a$.

$$
\begin{aligned}
& m=2 \pi\left(\frac{2 f}{\rho 10^{9}}\right)^{1 / 2}=\frac{\sqrt{2}}{\rho} \\
& g(x)=\frac{\rho(x)}{\rho_{0}}
\end{aligned}
$$

$\rho_{0}$ étant la résistivité nominale.

Les solutions de l'équation différentielle ont été obtenues pour un certain nombre de fonctions $g(x)$ dont les paramètres ont été déterminés par des calculs de statistique effectués par un ordinateur de façon à obtenir le meilleur lissage des courbes expérimentales.

Cet exemple constitue l'un des seuls mettant en évidence par la méthode des courants de Foucault les différentes valeurs de la conductivité entre une profondeur de métal comprise entre $300 \mu$ et $3 \mu$ (Fig. 2). 


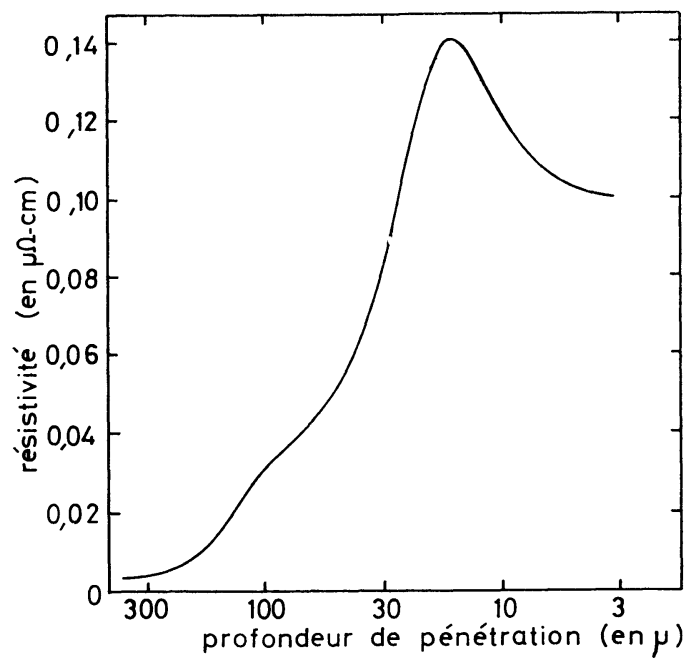

FIG. 2. - Variation de la résistivité d'un échantillon de cuivre en fonction de la profondeur de pénétration [8].

Enfin, ce type de méthode a également été utilisé pour mesurer la résistivité résiduelle de matériaux supra-conducteurs, par Burger et Deutscher [10], [11], puis Lauriat et Perio [12] utilisant le même appareillage (Fig. 3) :

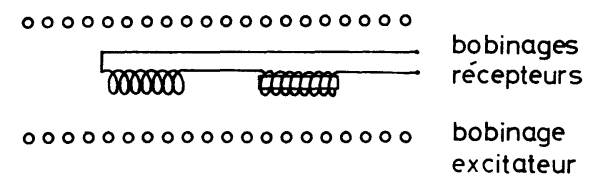

FIG. 3. - Coupe montrant la disposition de l'échantillon dans la bobine dans le cas de deux bobinages récepteurs montés en opposition [10], [11].

Le champ alternatif $H=H_{0} \mathrm{e}^{j \omega t}$ est produit par une première bobine, à l'intérieur de laquelle se trouvent deux bobinages récepteurs montés en opposition. En l'absence d'échantillon, ces bobines sont compensées de sorte que la tension mesurée aux bornes des bobines réceptrices est nulle. Ce dispositif permet de mesurer avec une bonne approximation des résistivités comprises entre $15 \times 10^{-6} \Omega . \mathrm{cm}$ et $10^{-8} \Omega . \mathrm{cm}$. Ce domaine pourrait être étendu vers des résistivités plus élevées grâce à une meilleure compensation des bobines.

Ces différentes techniques « statiques » permettent donc de mesurer des résistivités sans qu'aucun contact électrique ne soit nécessaire, ce qui est particulièrement intéressant pour des échantillons corrosifs ou des échantillons de très haute pureté pour lesquels une soudure de contact fausserait les résultats.

Cependant, grâce à l'effet de peau, ces méthodes permettent, dans certaines conditions de température et de fréquence, de mesurer la conductivité de surface et de mettre en évidence l'influence du mode de préparation et de l'état de surface sur la conductivité.

2.3 Méthodes DYNAMiQues. - On a appelé les méthodes décrites dans ce paragraphe «dynamiques » car elles consistent à étudier des phénomènes transitoires tels l'amortissement des courants induits dans l'échantillon lorsque le champ inducteur varie brusquement ou l'amortissement du mouvement de l'échantillon dans un champ magnétique. Dans les deux cas, il est relativement aisé de calculer la résistivité en fonction des grandeurs mesurées, s'il s'agit d'échantillons de forme simple, cylindrique ou sphérique.

La valeur de la profondeur de pénétration est alors définie à partir de la vitesse de la variation du flux, déterminée soit par le temps de montée de l'impulsion, soit par l'amortissement du mouvement de l'échantillon dans le champ magnétique. Il faut noter que si dans la mesure de l'amortissement des courants induits, la fréquence correspondante au temps de montée peut atteindre $1 \mathrm{MHz}$, dans le second cas, cette fréquence est plus faible et la résistivité mesurée est pratiquement la résistivité de volume.

2.3.1 Mesure de la décroissance des courants de Foucault (réf. [16] à [24]). - Un premier exemple de mesure de résistivité à partir de la mesure de la constante de temps de décroissance des courants de Foucault est celui de l'expérience de Baird et Boyle [17]: l'échantillon a la forme d'une spire de cuivre de $4 \mathrm{~cm}$ de diamètre et de $0,2 \mathrm{~cm}$ d'épaisseur. Le champ inducteur est produit par une bobine parcourue par un courant pulsé. Le temps de relaxation des courants induits dans la spire est mesuré à l'oscilloscope. Cette méthode cependant exige une forme d'échantillon particulière et ne donne qu'une valeur moyenne de la résistivité.

Une méthode plus générale a été décrite par Bean, de Blois et Nesbitt [18]. L'échantillon est placé dans une bobine inductrice (Fig. 4) à laquelle on applique

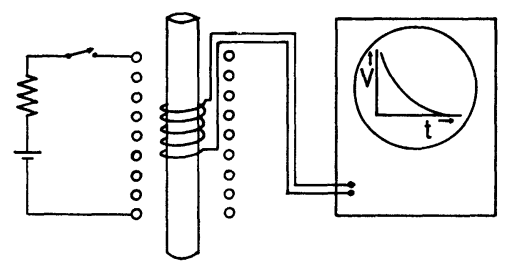

FIG. 4. - Schéma du dispositif permettant la mesure du temps de décroissance des courants de Foucault lorsque le champ magnétique varie [18].

une impulsion de courant rectangulaire. Un bobinage récepteur de même axe permet de mesurer la tension induite, directement relevée en fonction du temps sur l'écran d'un oscilloscope. Une valeur approchée de la fonction $V(t)$ a été calculée pour un échantillon cylindrique :

$$
V(t) \rightarrow 10 N \rho H_{0} \exp \left[-\frac{t}{\tau_{R}}\right]
$$

avec

$$
\tau_{R}=\frac{2,7 \mu R^{2} 10^{-9}}{\rho}
$$


et pour un échantillon parallélépipédique :

$$
V(t) \rightarrow \frac{160 N \rho}{\pi^{3}} H_{0} \frac{a^{2}+b^{2}}{a b} \exp \left[-\frac{t}{\tau_{a b}}\right]
$$

avec

$$
\tau_{a b}=\frac{1,27 \mu a^{2} b^{2} 10^{-9}}{\rho\left(a^{2}+b^{2}\right)} .
$$

Dans ces expressions :

$N$ nombre de tours de la bobine réceptrice,

$\rho \quad$ résistivité de l'échantillon en $\Omega$.cm,

$H_{0}$ champ magnétique maximum,

$R$ rayon du cylindre, en $\mathrm{cm}$,

$a, b$ dimensions transversales du barreau parallélépipédique en $\mathrm{cm}$.

Cette méthode est appliquée à l'étude de la résistivité à $4,2^{\circ} \mathrm{K}$ d'un échantillon cylindrique de tantale en fonction du degré de purification du métal.

Mordike et coll. [20], [21] ont utilisé pour leur part une méthode basée sur la mesure du rapport du temps de décroissance des courants de Foucault à deux températures différentes, dans des métaux de haute pureté. Cette méthode est ainsi indépendante de la géométrie de l'échantillon mais ne donne que des indications sur les impuretés contenues dans le métal.

Cependant, Le Page et coll. [22], analysant ces méthodes de mesures de décroissance des courants de Foucault, en soulignent les difficultés et les erreurs que peut entraîner une mauvaise interprétation des résultats. Il s'agit tout particulièrement de bien déterminer les constantes de temps du circuit de mesure avant de pouvoir affirmer que les valeurs mesurées sont caractéristiques de l'échantillon et peuvent être reliées à sa résistivité par les relations établies par Bean et coll. [18].

Enfin, J. E. Neighbor [19] a généralisé la méthode au cas où la résistivité est anisotrope. Il est en particulier possible de déterminer le tenseur de résistivité à partir des mesures du temps de décroissance des courants de Foucault, pour différentes orientations cristallines de l'échantillon par rapport au champ inducteur.

2.3.2 Amortissement du mouvement d'un conducteur dans un champ magnétique (réf. [25] à [38]). - Cette méthode consiste à étudier le mouvement d'un solide conducteur dans un champ magnétique. Pour cela, il y a essentiellement deux façons de procéder, l'échantillon constituant un pendule :

- l'échantillon est animé d'un mouvement pendulaire au départ en l'absence de champ magnétique. Un champ magnétique uniforme induit des courants dans le métal, entraînant un amortissement du mouvement ;

- l'échantillon est immobile en l'absence de champ ; un champ magnétique tournant lui communique un couple mécanique.

Ces méthodes ont été beaucoup utilisées, et depuis longtemps à en croire les références citées par Colin [25] [26] à [33]. Himsted [30], Lamb [31], Marcus [32], Keeping [33] se sont penchés sur l'aspect théorique du problème et ont donné des solutions pour des solides cylindriques et sphériques.

Colin [25], [34] a conçu un appareillage permettant la mesure de la résistivité et de l'anisotropie magnétique de composés d'insertion du graphite : l'échantillon est suspendu à un fil de torsion et placé dans un champ magnétique uniforme, homogène. En l'absence de champ, l'équation générale du mouvement est la suivante :

$$
I \frac{\mathrm{d}^{2} \theta}{\mathrm{d} t^{2}}+F_{0} \frac{\mathrm{d} \theta}{\mathrm{d} t}+C_{0} \theta=0
$$

Si on peut admettre que le moment des forces dues aux courants induits en présence d'un champ magnétique $H$ est proportionnel à la vitesse, cette équation se transforme en :

$$
I \frac{\mathrm{d}^{2} \theta}{\mathrm{d} t^{2}}+F_{1} \frac{\mathrm{d} \theta}{\mathrm{d} t}+C_{0} \theta=0
$$

De plus, si l'échantillon présente une anisotropie magnétique, il apparaît un couple supplémentaire $C_{\mathrm{m}}$ ou " constante de torsion magnétique " s'ajoutant au couple $C_{0}$.

Dans ces expressions :

$I$

$F_{0}(\mathrm{~d} \theta / \mathrm{d} t)$

$F_{\mathrm{m}}=F_{1}-F_{0} \quad$ coefficient de frottement magnétique dû aux courants induits.

$C_{\mathrm{m}}=C_{1}-C_{0}$ constante de torsion due à l'anisotropie $\Delta \chi$ de la susceptibilité magnétique.

Pour des échantillons cylindriques de diamètre $a$ et d'épaisseur $e$ ne présentant pas d'anisotropie magnétique $\left(C_{\mathrm{m}}=0\right)$, la relation liant $F_{\mathrm{m}}$ à la résistivité $\rho_{\mathrm{m}}$ est la suivante :

$$
F_{\mathrm{m}}=\frac{\pi a^{4} e H^{2}}{8 \rho_{\mathrm{m}}} .
$$

La mesure de $F_{\mathrm{m}}$ obtenue à partir de celle des différents paramètres de l'équation différentielle permet de déterminer $\rho_{\mathrm{m}}$, valeur qui est différente de $\rho_{\mathrm{r}}$ mesurée par la méthode à quatre points lorsqu'il existe une anisotropie magnétique. Cette étude a été faite par les auteurs pour des échantillons métalliques ( $\mathrm{Pb}, \mathrm{Al}, \mathrm{Sn}$, laiton) et sur des pyrocarbones purs et des pyrocarbones ayant subi une bromation " in situ ".

Le deuxième type de méthode, consistant à mesurer le couple mécanique auquel est soumis l'échantillon 
dans un champ magnétique tournant, a été utilisé par de nombreux expérimentateurs. Regel [35] par exemple, a mis au point un dispositif permettant d'étudier la variation de la résistivité de l'indium en fonction de la température. La sphère d'indium est suspendue par un fil de torsion, le champ magnétique tournant est produit par trois bobines disposées à $120^{\circ}$ l'une de l'autre. Le moment du couple mesuré est relié à la perte d'énergie par courants induits, donc à la résistivité. La valeur de la conductivité de différents métaux à des températures supérieures à $1250{ }^{\circ} \mathrm{C}$ a été obtenue avec une précision de $1 \%$ par Roll et Felger [36] par une expérience semblable. Dans une publication récente, Chaberski [36a] a étudié théoriquement la relation entre la résistivité et le couple auquel est soumis un échantillon conducteur placé dans un champ magnétique tournant, et décrit un dispositif permettant la mesure des résistivités dans un large domaine $\left(10^{-8} \Omega . \mathrm{cm}\right.$ à $\left.10^{8} \Omega . \mathrm{cm}\right)$. Une variante de cette méthode a été décrite par Ramanathan et Dhillon [37] ; ces auteurs ont mesuré l'impulsion mécanique communiquée à l'échantillon par un brusque changement du champ magnétique.

Enfin, il faut décrire une méthode originale qui, en réalité, ne peut être rattachée ni aux méthodes statiques, ni aux méthodes dynamiques de façon rigoureuse, mais qui constitue plutôt un cas intermédiaire. Le dispositif mis au point par Haisty [38] est le suivant (Fig. 5) : l'échantillon, contenu dans

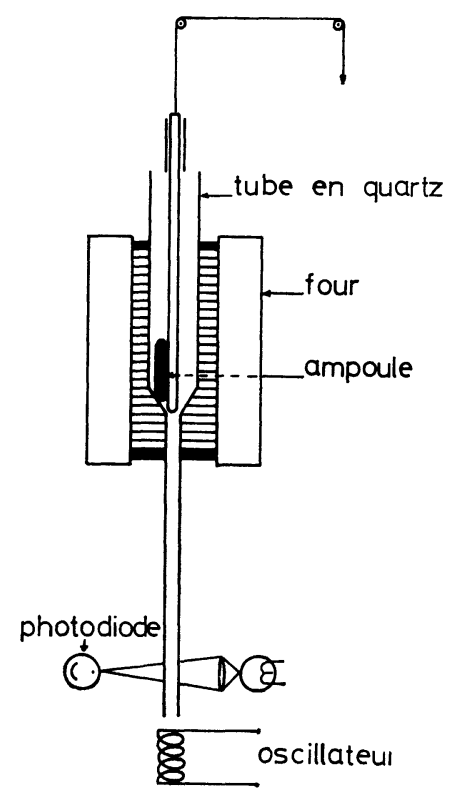

FIG. 5. - Schéma du dispositif décrit par Haisty [38].

une ampoule en quartz tombe, guidé par un tube en quartz également, à travers la bobine d'un oscillateur Collpitts résonnant à $14 \mathrm{MHz}$. Les courants induits dans l'échantillon par son passage dans le champ HF de la bobine entraînent une variation de la charge de l'oscillateur qui se traduit par une variation du courant grille. La courbe donnant la valeur de ce courant en fonction de la résistivité a été tracée à partir d'échantillons étalons. La variation de ce courant donne sur l'écran d'un oscilloscope une impulsion dont la forme permet de mettre en évidence des inhomogénéités de l'échantillon. Cette méthode offre un certain nombre d'avantages sur les méthodes précédemment décrites : d'une part la gamme de résistivités mesurables est étendue et recouvre un domaine allant de $10^{-5} \Omega$.cm à $10^{8} \Omega$.cm; d'autre part, un four situé avant le tube de guidage permet de porter les échantillons à des températures élevées (en vue de l'étude de solides en fusion), et le temps de passage entre la sortie du four et la bobine étant trop bref, il n'y a pratiquement pas de variation de température. Un dispositif semblable pourrait d'ailleurs être envisagé pour les mesures de résistivité à basse température.

2.4 Conclusion. - Les différentes méthodes de mesure de conductivité par courant de Foucault, qu'on a brièvement décrites dans les paragraphes précédents, permettent ainsi, d'une part, d'éliminer des mesures, le problème des contacts électriques qui souvent sont technologiquement difficiles et qui, de plus, sont une cause d'erreur considérable dans la détermination de la résistivité ; d'autre part, ces méthodes permettent, dans certains cas, d'obtenir des renseignements sur la conductivité de surface.

Cependant, en général, la profondeur de pénétration est encore importante, dépassant largement les couches superficielles et seules les mesures en hyperfréquence sont réellement intéressantes pour étudier les propriétés électriques des surfaces solides.

3. Mesures de conductivité en hyperfréquence. Le développement des techniques hyperfréquence a conduit à examiner de façon approfondie les propriétés de conduction des matériaux utilisés dans les guides d'onde ou les cavités résonnantes.

En effet, la théorie de la propagation des ondes électromagnétiques dans les circuits hyperfréquence est basée sur l'hypothèse d'une conductivité infinie des parois métalliques. En réalité, cette conductivité a une valeur finie et il y a ainsi amortissement de l'onde par perte d'énergie Joule dans les parois; à cause de l'effet pelliculaire, seule une très faible épaisseur du métal y contribue, et l'état de surface des parois joue un rôle primordial. Il est donc essentiel de bien connaître les propriétés électriques superficielles des parois des guides d'ondes ou des cavités résonnantes afin de minimiser dans le premier cas l'atténuation de l'onde, dans le second cas, la diminution du coefficient de surtension.

Les mesures de conductivité de surface en hyperfréquences sont de deux sortes :

- la mesure de l'atténuation d'une onde dans un guide ;

- la mesure du coefficient de surtension d'une cavité résonnante. 
Il a été fréquemment observé que la valeur de la conductivité ainsi mesurée était inférieure à la valeur déterminée en courant continu. Ceci montrait la grande importance de la préparation de la surface. La question de l'influence de l'état de surface sur la conductivité sera discutée dans le prochain chapitre alors que, dans ce qui suit, on se bornera à décrire et analyser comme précédemment, les différentes techniques.

3.1 GuIDES D'ONDES (RÉFÉRENCES [39] A [41]). L'atténuation d'une onde électromagnétique se propageant dans un guide est due essentiellement aux pertes par effet Joule dans les parois et dépend donc directement de la résistivité de surface. Si le guide est court-circuité, la valeur du taux d'ondes stationnaires (TOS) est une fonction connue de la conductivité. Soit $s$ le TOS mesuré à l'entrée du guide; le coefficient d'atténuation, exprimé en décibels est :

$$
\alpha=\frac{10}{l} \log \frac{s+1}{s-1} .
$$

Dans le cas particulier d'un guide rectangulaire fonctionnant dans le mode $\mathrm{TE}_{10}$ [39], ce coefficient est relié à la conductivité par la relation suivante :

$$
\begin{array}{r}
\alpha=\frac{1}{2 b} \frac{1}{\left[1-\left(\frac{\lambda_{0}}{\lambda_{c}}\right)^{2}\right]^{1 / 2}}\left(\frac{4 \pi}{\lambda_{0} \mu c \sigma}\right)^{1 / 2} \times \\
\times\left[1+\frac{2 b}{a}\left(\frac{\lambda_{0}}{\lambda_{c}}\right)^{2}\right] .
\end{array}
$$

Dans cette expression :

$a$
$b$
$\lambda_{0}$
$\lambda_{\mathrm{c}}=2 a$
$\mu_{0}=4 \pi \times 10^{-7} \mathrm{H} / \mathrm{m}$
$c$

Les parois du guide sont constituées par le métal échantillon, dans cette expérience décrite par Maxwell [39]. La longueur $l$ du guide est choisie de façon à obtenir une valeur du TOS de 5 ou 6 . Les métaux étudiés sont le cuivre, l'aluminium, le nickel, l'argent, l'or, etc...

Les valeurs des conductivités obtenues par cette méthode et par la méthode de la cavité résonnante dont on reparlera, sont en général sensiblement plus faibles que les conductivités mesurées en courant continu.

Une méthode semblable est décrite par Chatterjee et coll. [40]. L'échantillon est une plaque courtcircuitant le guide. On détermine alors le coefficient de réflexion $\rho$ de la plaque, relié à la conductivité par la relation :

$$
\sigma=\frac{\pi}{\mu f \lambda_{g}(1-\rho)^{2}}
$$

où $\lambda_{g}$ est la longueur d'onde caractéristique dans le guide. Pour cela, on mesure le TOS en un point proche de la terminaison, par la méthode de la phase ou par une méthode de Hipple modifiée [41]. La précision de la mesure de $\sigma$, fonction de l'erreur expérimentale sur $\lambda_{g}, f, \rho$, est estimée à $3 \%$. Les valeurs des conductivités de surface de cuivre, d'argent, de laiton ainsi obtenues sont légèrement inférieures aux valeurs obtenues par Maxwell. Les valeurs expérimentales des coefficients d'atténuation $\alpha$ sont plus élevées que les valeurs théoriques calculées d'après la relation précédente. Cette différence peut être attribuée à l'état de surface des parois du guide et surtout de l'échantillon court-circuitant le guide d'onde. Les auteurs soulignent d'ailleurs qu'ils n'ont pas tenu compte de l'erreur introduite de ce fait dans la détermination de $\sigma$, erreur particulièrement sensible dans le cas du cuivre qui se recouvre, à l'air, d'une couche d'oxyde altérant de façon importante la valeur de la conductivité.

3.2 CAVItÉS RÉSOnNANTES. - Cette deuxième méthode de détermination des conductivités de surface est, de beaucoup, la plus couramment utilisée, les résultats obtenus étant généralement plus précis. Elle consiste à mesurer le coefficient de surtension d'une cavité résonnante fonctionnant dans un mode donné. Par définition, le coefficient de surtension $Q$ est déterminé par le rapport de l'énergie électromagnétique emmagasinée et de l'énergie thermique dissipée par effet Joule dans les parois, par période :

$$
Q=2 \pi f \frac{E_{\text {emm. }}}{E_{\text {diss. }}} .
$$

D'autre part, on a défini l'impédance de surface dans le premier paragraphe :

$$
Z_{\mathrm{s}}=R_{\mathrm{s}}+j X_{\mathrm{s}}=\left(\frac{E_{x}}{H_{y}}\right)_{z=0}
$$

et on a montré que $R_{\mathrm{s}}=1 / \sigma \delta$. Le coefficient de surtension peut alors s'écrire :

$$
Q=\frac{\pi\left(\mu_{0} / \varepsilon_{0}\right)^{1 / 2}}{R_{\mathrm{s}}}\left[\frac{2}{\lambda} \frac{\iiint_{\mathrm{v}} H^{2} \mathrm{~d} v}{\iint_{\mathrm{s}} H^{2} \mathrm{~d} s}\right] .
$$

La quantité entre crochets est une constante pour une cavité fonctionnant dans un mode donné : d'où

$$
Q=\frac{A \pi\left(\mu_{0} / \varepsilon_{0}\right)^{1 / 2}}{R_{\mathrm{s}}}=A c\left(\pi \mu_{0}\right)^{1 / 2}\left(\frac{\sigma}{f}\right)^{1 / 2} .
$$


On ne s'étendra pas longuement sur les différentes méthodes de mesure de coefficient de surtension, qui sont décrites dans les ouvrages généraux [43], [44], [45], [46], [47]. Il faut cependant souligner que la précision de la détermination de $\sigma$ est d'autant meilleure que le coefficient de surtension est élevé. Cependant, les mesures précises de $Q$ élevés sont délicates. Les méthodes décrites par Salaün [46] permettent d'atteindre une précision de $5 \%$ sur des coefficients de surtension de $10^{4}$ à $10^{7}$.

Il existe un grand nombre de références concernant les mesures de résistances et d'impédances de surface. Dans le cadre de cette étude, nous n'en avons pas fait la bibliographie exhaustive ; nous donnons quelques références [48] à [54] en détaillant cependant les méthodes les plus caractéristiques.

Il y a essentiellement deux positions possibles de l'échantillon dans la cavité :

- il peut constituer une face amovible de la cavité ;

- le métal dont on étudie les propriétés de conductivité de surface constitue la cavité elle-même.

Maxwell [39] décrit dans l'article précédemment cité, une méthode de mesure de conductivité de surface à l'aide d'une cavité résonnante. Plusieurs types de cavités sont envisagées : une cavité cylindrique fonctionnant dans le mode $\mathrm{TE}_{011}$, l'échantillon formant l'une des faces terminales du cylindre, une cavité coaxiale (TEM) pour laquelle l'échantillon constitue le conducteur central, des cavités rectangulaires différemment assemblées.

Des cavités rectangulaires ou cylindriques à fond plat ont généralement été choisies pour les expériences car elles offraient le meilleur compromis entre la forme de l'échantillon (la plus simple possible à cause du polissage) et la simplicité de la mesure. Ces cavités fonctionnent dans le mode $\mathrm{TE}_{10}$ et la valeur du coefficient de surtension est obtenue à partir du tracé de la bande passante déterminée par mesure du TOS. Lorsqu'on remplace le fond d'une cavité résonnante par un échantillon, le nouveau coefficient de surtension s'obtient aisément de la façon suivante [46], [55] : le coefficient de surtension est égal (à un facteur peu différent de 1 près) au rapport du volume de la cavité au volume compris dans l'épaisseur de peau. Ce facteur dépend du mode choisi et il est le même avec ou sans échantillon :

- soit $Q_{1}$ le coefficient de surtension de la cavité avec un fond métallique pour lequel, à la fréquence choisie, la profondeur de pénétration est $\delta_{1}$ :

$$
Q_{1}=a \frac{V}{S \delta_{1}} .
$$

- Si on remplace le fond métallique par l'échantillon dans lequel la profondeur de pénétration est $\delta_{2}$, on obtient un coefficient de surtension $Q_{2}$

$$
Q_{2}=a \frac{V}{(S-s) \delta_{1}+s \delta_{2}}
$$

volume de la cavité,

surface totale de la cavité,

surface du fond de la cavité.

Ce principe a été utilisé par Salaün [46] dans un dispositif à barillet permettant d'étudier successivement six échantillons dans les mêmes conditions; la cavité cylindrique $\left(\mathrm{TE}_{01 \mathrm{n}}\right)$ se compose de trois parties : un corps cylindrique, le fond supérieur muni des dispositifs de couplage, le fond inférieur interchangeable sur lequel sont évaporés les métaux supraconducteurs $(\mathrm{Pb}, \mathrm{Nb})$ à étudier. La cavité résonnant dans le mode $\mathrm{TE}_{01 \mathrm{n}}$, les lignes de courant ne coupent pas les régions de contact entre les différentes parties, ce qui minimise les erreurs dans la détermination de $\sigma$.

La valeur de la conductivité de surface est particulièrement importante dans les applications des hyperfréquences, plus spécialement dans les accélérateurs linéaires haute énergie où la puissance perdue par effet Joule dans les parois du guide accélérateur est importante. Afin de réduire les pertes, Biquard et Septier [56] ont étudié la résistance superficielle de cuivre et d'aluminium à différentes températures $\left(77^{\circ} \mathrm{K}, 20,4^{\circ} \mathrm{K}, 4,2^{\circ} \mathrm{K}\right)$. Des cavités de forme simple en cuivre ou en aluminium ont été étudiées :

- une cavité cylindrique résonnant dans le mode $\mathrm{TE}_{011}$ à $3160 \mathrm{MHz}$,

- deux cavités coaxiales (TEM) résonnant à $3160 \mathrm{MHz}$ et $1420 \mathrm{MHz}$.

Ces auteurs ont d'ailleurs plus particulièrement étudié l'influence du mode de préparation de la surface sur le facteur de surtension d'une cavité en cuivre, $\mathrm{TE}_{011}$ résonnant à $3160 \mathrm{MHz}$ : en (a) il s'agit d'une cavité à parois polies électrolytiquement, en $(b)$ d'un polissage mécanique et en $(c)$ d'une surface brute d'outil. Cette courbe montre également une influence beaucoup plus grande de l'état de surface à basse température (Fig. 6).

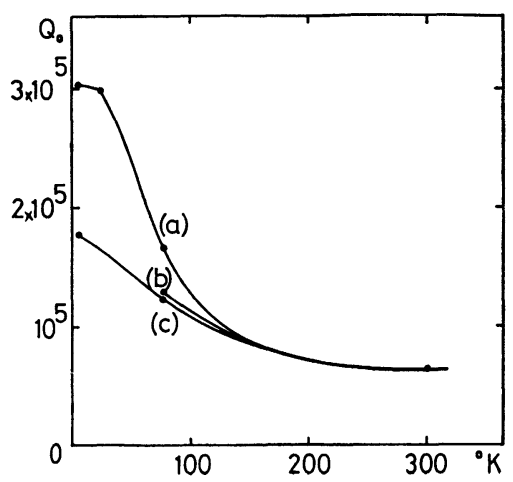

Fig. 6. - Variation du facteur de surtension d'une cavité résonnante en fonction de la température pour des états de surface de parois différents : $a$ ) surface polie électrolytiquement, b) surface polie mécaniquement, c) surface brute d'usinage.

3.4 INFLUENCE DE L'ÉTAT DE SURFACE SUR LA CONDUCTIVITÉ EN HYPERFRÉQUENCES. - Des différentes 
expériences décrites précédemment, il faut retenir ceci : la mise en évidence d'une différence fondamentale entre conductivité de surface et conductivité de volume ne peut se faire que sur une surface parfaitement lisse. Ce cas idéal n'étant jamais atteint, il faut essayer de minimiser les irrégularités. Morgan [57] a étudié théoriquement l'influence de certaines irrégularités de surface de forme définie, sur la puissance dissipée par les courants de Foucault sur une surface métallique. Citons un exemple: dans le cas d'une surface présentant des rainures dont la direction moyenne est perpendiculaire à celle des courants induits et dont la taille moyenne est comparable à la profondeur de pénétration, la puissance dissipée peut être augmentée de $60 \%$ de la puissance dissipée sur une surface parfaitement lisse. Dans le cas où ces rainures sont dirigées parallèlement à la direction du courant induit, on ne note qu'une augmentation de $20 \%$ de la puissance dissipée sur une surface lisse.

Bien que la distribution des rainures sur une surface soit aléatoire et donc que leur effet correspondant a une valeur intermédiaire comprise entre ces deux extrêmes, ces chiffres donnent un ordre de grandeur de l'influence des défauts de surface se répercutant sur la détermination de conductivités de surface par une augmentation de la valeur de $\sigma$.

De plus, cet effet, auquel s'ajoute à basse température, l'effet de peau anormal, complique singulièrement l'interprétation des résultats obtenus et masque généralement l'existence d'une couche superficielle dont la conductivité intrinsèque serait plus faible. La préparation de la surface à étudier est donc primordiale. Chambers et Pippard [58] ont rassemblé dans un article de synthèse les résultats obtenus par différents auteurs sur l'influence de la préparation de la surface sur la conductivité.

Les surfaces polies électrolytiquement sont les plus lisses suivies par les surfaces polies mécaniquement, puis les surfaces brutes d'usinage. Cette étude a été faite au microscope par Biquard et Septier [56] dans un article précédemment cité. Ces auteurs ont également étudié l'influence de la composition du bain électrolytique sur l'état de surface de parois de cavités résonnantes en cuivre et en aluminium. Semblable étude a été faite par Salaün [46] pour des dépôts de plomb et de niobium. Les résistances de surface obtenues pour des échantillons électrodéposés sans additif organique sont élevées car il existe à la surface des reliefs importants et on observe une discontinuité entre grains. Par contre avec un additif organique, la taille des grains est plus faible et la résistance de surface, qui diminue également avec la densité de courant de dépôt électrolytique, est moins grande.

4. Application des méthodes de mesure de conductivités en courant alternatif à l'étude des propriétés électriques de poudres faiblement conductrices. 4.1 PROBLÈMES POSÉS PAR LES MESURES DE CONDUC-
TIVITÉS DE POUDRES. - Les nombreux travaux consacrés à l'étude de la conductivité de poudres faiblement conductrices ont mis en évidence la complexité du problème, due essentiellement à l'indétermination des résistances de contact particule-particule et poudre-électrode [59], [60]. C'est pourquoi de nombreux expérimentateurs ont pensé résoudre le problème en mesurant, en courant continu, la résistivité de la poudre comprimée sous forte pression $\left(100\right.$ à $\left.1000 \mathrm{~kg} / \mathrm{cm}^{2}\right)$. Ces auteurs admettent que l'échantillon comprimé est équivalent au monocristal, lorsque la conductivité de la poudre devient indépendante de la pression appliquée [61]. Kronick et Labes [62] ont montré que cette méthode conduisait à des résultats discutables dans le cas de substances anisotropes et Mrozowski [63] étudiant la résistivité de poudres de carbone fortement comprimées, souligne la difficulté d'interpréter physiquement les résultats expérimentaux. De fait, la plupart des auteurs [64] s'accordent à dire que les méthodes de mesure de résistivité, sous forte pression, donnent des résultats qualitatifs, masquant toute information concernant l'anisotropie des grains, la résistance interparticule et les effets de surface.

Les mesures de résistivité de poudres en courant alternatif permettent de résoudre un certain nombre de ces problèmes. En effet Parfit (cité par Eley [65]) a montré que, pour une fréquence supérieure à $6 \mathrm{MHz}$, la résistivité d'un échantillon d'isobenzanthrone restait constante, ce qui signifie qu'il existe un seuil de fréquence à partir duquel la résistance de contact entre grains est court-circuitée par sa propre capacité. Le schéma électrique équivalent d'une poudre faiblement comprimée [66] est obtenu en considérant que chaque grain possède une résistance $R_{\mathrm{s}}$ et une capacité $C_{\mathrm{s}}$. Le contact entre grains est représenté par une résistance $R_{\mathrm{c}}$ et une capacité $C_{\mathrm{c}}$. L'étude théorique du comportement d'une poudre faiblement conductrice en fonction de la fréquence se ramène à celle du schéma équivalent $R_{\mathrm{p}}, C_{\mathrm{p}}$, conduisant à l'élaboration de différents modèles [64], [66], [67], [68], [69].

Zeeck et Voitländer [70] ont montré que les résultats expérimentaux obtenus lorsque la conductivité devient indépendante de la fréquence, pouvaient être interprétés au moyen de la théorie de Maxwell-Wagner concernant les diélectriques inhomogènes. La conductivité mesurée dans ce domaine de fréquence peut être considérée comme la conductivité spécifique de la poudre.

4.2 Méthodes DE Mesures. - 4.2.1 Mesures en basse fréquence. - Le dispositif le plus simple et le plus couramment utilisé consiste à comprimer légèrement la poudre entre les deux électrodes de mesure [66]. Ce dispositif cependant ne résout pas le problème du contact poudre-électrode. Snowden et coll. [67], [71] ont décrit une cellule coaxiale dans laquelle est placé l'échantillon (oxyde de zinc); un tassement régulier de la poudre est obtenu par vibrations mécaniques 
de la cellule. La résistivité est mesurée au moyen d'un pont d'impédances, l'ensemble cellule-échantillon étant considéré comme un condensateur avec pertes. Cependant Ogawa [72] souligne l'intérêt des mesures de résistivités de poudres semiconductrices, ne nécessitant aucun contact électrique. Cet auteur décrit une méthode qui consiste à disperser la poudre dans une résine et à mesurer le couple mécanique auquel est soumis l'échantillon placé dans un champ électrique tournant. Le domaine de résistivités mesurables par cette méthode est large $\left(10^{3} \Omega . \mathrm{cm}\right.$ à $\left.10^{10} \Omega \mathrm{cm}\right)$.

Enfin, il existe différentes méthodes de mesures "statiques» de conductivité d'échantillons semiconducteurs massiques [73], [74], [75], [76]. Ces méthodes diffèrent de celles utilisées pour les échantillons bons conducteurs : dans le cas de substances de haute résistivité, la liaison inductive est remplacée par une liaison capacitive. Il n'est pas impossible d'envisager de telles mesures sur des poudres qui seraient dispersées dans une résine ou contenues dans une ampoule de quartz.

4.2.2 Mesures en hyperfréquence. - L'étude de la résistivité de poudres en hyperfréquence est généralement réalisée par la mesure de la variation du coefficient de surtension d'une cavité résonnante [66], [70], [77]. La poudre est placée soit sur le fond de la cavité [70], soit au centre de la cavité remplie partiellement d'une mousse synthétique dans laquelle est percé un trou de faible diamètre [77].

Zeeck et Voitländer [70] ont montré l'intérêt de ce type de mesure dans l'étude de la chimisorption de différents gaz sur une poudre semiconductrice (oxyde de zinc pur ou dopé, oxyde d'aluminium). Une méthode différente a été décrite par Snowden et coll. [71] : la cellule coaxiale utilisée en basse fréquence, est considérée en hyperfréquence comme une ligne de transmission ouverte, terminée par l'échantillon. Ce dispositif permet la mesure de conductivité de poudres dans un large domaine de fréquence $\left(10^{3} \mathrm{~Hz}\right.$ à $\left.10^{10} \mathrm{~Hz}\right)$.

4.3 Conclusion. - Les résultats des mesures de résistivités de poudres en courant alternatif, obtenus par les différents auteurs, montrent que l'interprétation théorique de la variation de la résistivité en fonction de la fréquence reste souvent délicate et ne permet pas, d'une manière générale, de tenir compte de tous les paramètres [64]. D'autre part, on a montré qu'il existait une fréquence seuil au-delà de laquelle la résistivité devenait constante, cette valeur pouvant alors être considérée comme la résistance spécifique de la poudre. Par conséquent, malgré les difficultés que présente l'interprétation des résultats, notamment dans le cas des poudres semiconductrices et le nombre relativement faible de travaux effectués dans ce domaine, il apparaît que les méthodes de mesure de résistivité de poudre en courant alternatif présentent un intérêt considérable.
Conclusion. - Cette étude bibliographique a permis d'examiner brièvement les diverses méthodes de mesure de résistivités en courant alternatif. Celles-ci ont été classées en deux groupes distincts : les mesures par courants de Foucault et les mesures en hyperfréquence. Les mesures par courants de Foucault sont préconisées généralement pour la détermination de la résistivité volumique de l'échantillon et présentent l'avantage, par rapport aux mesures en courant continu, de ne nécessiter aucun contact électrique, alors qu'en hyperfréquence, seule la résistivité de surface peut être obtenue. Cependant cette frontière, due à la variation de la profondeur de pénétration de l'onde électromagnétique dans l'échantillon, dépend essentiellement de la valeur de la résistivité à mesurer. En conclusion, on va donc rapidement examiner les différents domaines de résistivités.

a) Echantillons bons conducteurs $\left(\rho<10^{-5} \Omega . \mathrm{cm}\right)$. Il s'agit du cas le plus favorable aussi bien en ce qui concerne la détermination de résistivité volumique que superficielle. En effet, la résistivité volumique peut être obtenue par les différentes méthodes statiques ou dynamiques, à condition d'utiliser une fréquence suffisamment faible pour diminuer l'effet de peau. Inversement, il est possible en haute fréquence ou à basse température, de mettre en évidence l'influence de la zone superficielle sur la conductivité. Cependant, seules les mesures en hyperfréquence constituent une méthode assez fine de mesure de conductivité superficielle.

b) Echantillons de conductivité moyenne

$$
\left(10^{-5} \Omega . \mathrm{cm}<\rho<10 \Omega . \mathrm{cm} .\right.
$$

La plupart des méthodes statiques sont intéressantes dans un domaine allant de $10 \Omega . \mathrm{cm}$ à $10^{-8} \Omega . \mathrm{cm}$ et sont donc encore applicables dans ce cas ; cependant la profondeur de pénétration reste grande, même en haute fréquence. S'il est aisé de mesurer des résistivités volumiques, il n'en est pas de même pour la conductivité superficielle. C'est le cas par exemple du graphite $\left(\rho_{\|} \simeq 10^{-5} \Omega . \mathrm{cm}\right)$ dont les propriétés électriques superficielles parallèlement aux plans graphitiques ne peuvent être déterminées qu'en hyperfréquence.

c) Echantillons faiblement conducteurs. - L'utilisation du couplage capacitif de l'échantillon au circuit de mesure permet l'extension des méthodes statiques à la détermination des hautes résistivités. D’autre part, en ce qui concerne les méthodes dynamiques, la valeur de la résistivité peut être obtenue par la mesure du couple auquel est soumis l'échantillon placé dans un champ électrique ou magnétique tournant.

Nous avons montré que ces méthodes pouvaient également être utilisées dans l'étude des propriétés électriques de poudres faiblement conductrices et nous avons souligné l'intérêt que présentaient les mesures de résistivité de poudre en hyperfréquence 
dans la mise en évidence de modifications chimiques superficielles (adsorption par exemple).

Par ailleurs, il faut noter que l'étude des propriétés électriques superficielles d'échantillons massiques faiblement conducteurs peut être entreprise par des méthodes différentes, qui n'ont pas été abordées car sortant du cadre de cet exposé : il s'agit des méthodes de réflexion d'ondes de fréquences plus élevées atteignant le domaine optique, telles l'électroréflectance [78] ou l'ellipsométrie [79].

Remerciements. - L'auteur tient à remercier M. J. Conard pour l'intérêt qu'il a porté à ce travail et les conseils qu'il lui a donnés lors de la rédaction.

\section{Bibliographie}

[1] Maxwell (J. C.), A treatise on electricity and magnetism, $3^{\mathrm{e}}$ édition, Dover, New York (1954).

[2] LondON (H.), H. F. Resistance of superconducting tin, Proc. Roy. Soc., 1940, A 176, 522.

[3] Pippard (A. B.), The anomalous skin effect in normal metals, Proc. Roy. Soc., 1947, A 191, 385.

[4] Reuter (G. E. H.), Sondheimer (E. H.), The theory of the anomalous skin effect in metals, Proc. Roy. Soc., 1948, A 195, 336. Nature, 1948, 169, 394.

[5] Rorschach (H. E.), Herlin (M. A.), Phys. Rev., $1951,81,467$.

[6] VAN DeR Berg (G. J.), VAn den Marel (L. C.), cité par GerRITSEN (A. N.), dans Encyclopedia of Physics, S. Flügge, Vol. XIX, 137 (Springer Verlag, Berlin, 1956).

[7] Zimmerman (J. E.), Measurement of electrical resistivity of bulk metal, Rev. Sci. Inst., 1961, 32, 402.

[8] Johnson (E. W.), Johnson (H. H.), Technique for measuring position dependent electrical resistivity, Rev. Sci. Inst., 1964, 35, 1510.

[9] HaGue (B.), Alternating current bridge methods, Sir Isaac Pitman and Sons Ltd (London 1957), p. 600 .

[10] Deutscher (G.), Mesure de résistivités par effet de peau normal Thèse $3^{\mathrm{e}}$ cycle, Faculté des Sciences, Orsay.

[11] Burger (J. P.), Deutscher (G.), Mesures de résistivités par effet de peau normal, J. Physique (Physique Appliquée), 1965, 26, 177 A.

[12] Lauriat (J. P.), Perio (P.), Dispositifs pour mesure de résistivités électriques entre $5^{\circ} \mathrm{K}$ et $100^{\circ} \mathrm{K}$, Rev. Phys. Appl., 1968, 3, 185.

[13] Hellenthal (W.), Lucke (R.), Ostholt (H.), Observation of spatially inhomogenous resistivity distributions using the skin effect at various frequencies, Zeit. für Angew. Phys., 1969, 26, 219.

[14] Daybell (M. D.), Eddy current method for low temperature resistivity measurements, Rev. Sci. Inst., 1967, 38, 1412.

[15] Deisinger (H.), HACKL (A. E.), Electrodeless measurements of electrical conductivity, Allg. Prakt. Chem., 1968, 19, 229.

[16] VoIGT (H.), Method for resistivity measurements without contacts, Zeit. für Angew. Phys., 1968, 25, 146.

[17] Baird (D. C.), BoYle (W. S.), Determining electrical resistivities at low temperatures, J. Appl. Phys., 1953, 24, 958.

[18] Bean (C. P.), de Blois (R. W.), Nesbitt (L. B.), Eddy current method for measuring the resistivity of metals, J. Appl. Phys., 1959, 30, 1976.

[19] Neighbor (J. E.), Eddy current method for measuring anisotropic resistivity, J. Appl. Phys., 1969, 40, 3078

[20] Clarke (J. M.), Mordike (B. L.), Continuous contact less resistivity measurements, Appl. Mater. Res. (G. B.), 1966, 5, 181.
[21] Mordike (B. L.), Balchin (N. C.), Contact free residual resistivity measurements on zone refined tantalum, Zeit. Metallk., 1963, 54, 272.

[22] Le Page (J.), Bernalte (A.), Lindholm (D. A.), Analysis of resistivity measurements by the eddy current decay method, Rev. Sci. Inst., 1968, 39, 1019.

[23] Grube (G.), Speidel (H.), The electrodeless measurement of electrical resistance of metals and alloys at high temperature, I. The electrical resistance of manganese, $Z$. Elektrochem. 1940, 46, 233.

[24] Roll (A.), Motz (H.), Electric resistance of metallics melts ; I. Method of measurement and electrical resistance of molten pure metals, Zeit. Metallk., 1957, 48, 272.

[25] Colin (G.), Setton (R.), Mesure de la résistivité électrique par courants induits, I. Etude et mise au point de la méthode, J. Chim. Phys., 1967, 64, 287.

[26] Weber, Ann. Phys., 1899, 68, 705.

[27] Davies (W. J.), Evans (E. J.), Electric conductivity of dilute $\mathrm{Na}$ amalgams at various temperatures, Phil. Mag., 1930, 7, 1.

[28] Dutta, Chowdhury, Ind. J. Phys., 1954, 28, 312.

[29] Baveja (K. D.), Electrical resistivities of metals by the method of magnetic damping, J. Sci. Ind. Res., 1961, 20B, 343.

[30] Himsted, Ann. der Phys., 1880, 11, 812.

[31] LaMB, Phil. Trans., 1883, 174, 519.

[32] Marcus, Phys. Rev., 1924, 24, 68.

[33] Keeping, Phil. Mag., 1930, 7, 16.

[34] Colin (G.), Mazières (C.), Mesures de résistivités par courants induits, II. Problèmes posés par l'anisotropie magnétique ; III. Etude " in situ » de l'interaction brome-pyrocarbone, J. Chim. Phys., 1970, 67, 323.

[35] Regel (A. R.), Zhur. Tekh. Fiz 1948, 18, 511.

[36] Roll (A.), Felger (H.), Motz (H.), Electrodeless measurements of the electrical conductivity by a rotary field method Zeit. Metallk., 1956, 47, 707.

[36a] Chaberski (A. Z.), Contactless induction method for electric resistivity measurement. J. Appl. Phys., 1971, 42, 940.

[37] Ramanathan (K. G.), Dhillon (J. S.), Nature, 1955, 175, 948.

[38] HaISTY (R. W.), Electrodeless measurement of resistivities over a very wide range, Rev. Sci. Inst., 1967, 38, 262.

[39] MaXwell (E.), Conductivity of metallic surfaces at microwave frequencies, J. Appl. Phys., 1947, $18,629$.

[40] Chatterjee (S. K.), Ramdas Shenoy (P.), Rama BaI (C.), A method for the measurement of conductivity of metals at microwave frequencies, $J$. Indian Inst. Sci., 1954, 36B, 107.

[41] Buchanan (T. J.), Proc. I. E. E., 1952, part 4, 99, 372. 
[42] Saito (S.), Suda (T.), Measurement of surface losses of metals plates and attenuation in a rectangular waveguide at $4000 \mathrm{MHz}, J$. Inst. Electr. Comm. Engrs. Japan, 1952, 35, 251.

[43] Montgomery (C. G.), Technique of microwave engineering, Mc Graw Hill Book Co. (New York, 1947).

[44] Harvey (A. F.), Microwave engineering, Academic Press (London, New York, 1963).

[45] Leibrecht (K.), New method for the measurement of the Q factor of cavities and its applications to research in solid state physics, Rev. Sci. Inst., 1968, 39, 1919.

[46] Salaün (M.), Détermination de coefficients de surtension très élevés. Application à la mesure en UHF de la résistance superficielle de matériaux supraconducteurs, Thèse de Docteur-Ingénieur (Orsay, 1969).

[47] Dagg (I. R.), Muc (A. M.), Reesor (G. E.), An accurate method for determining $Q$ factor of a resonant cavity, Canad. J. Phys., 1968, 46, 1413.

[48] DöRING (H.), KLEIN (W.), Resonance resistance measurements at centimetric wavelenghts, Arch. Tech. Messen, 1951, 191, T 135.

[49] Emeleus (T. G.), Mc Clausland (M. A. H.), Surface resistance and effective permeability of rare earth metals at microwave frequencies, J. Phys. $C$ (Solid State Physics), 1969, ser. 2, 2, 1867.

[50] FischeR (G.), MAKI (K.), High frequency surface impedance of dirty type, II. supraconductors in the surface sheat regime Phys. Rev., 1968, 176, 58.

[51] Garfunkel (M. P.), Surface impedance of type I supraconductors: calculation of the effect of a static magnetic field, Phys. Rev., 1968, 176, 516.

[52] Gilchrist (J. G.), Microwave surface resistance of type II supraconductors, Proc. Roy. Soc., 1966, A 295, 399.

[53] Hahn (H.), Halama (H. J.), Foster (E. H.), Measurements of the surface resistance of superconducting lead at 2,868 GHz, J. Appl. Phys., 1968, 39, 2606.

[54] SERIN (B.), The conductivity of metals at microwave frequencies, Phys. Rev., 1947, 72, 1261.

[55] Conard (J.), Mesure par effet de peau de la désorganisation du réseau du graphite au voisinage de la surface, CNRS, RCP No3, rapport GFEC. (Février 1966).

[56] Biquard (F.), Septier (A.), Amélioration de la conductivité superficielle du cuivre et de l'aluminium en hyperfréquences par abaissement de température, Nucl. Inst. Meth., 1966, 44, 18.

[57] Morgan (S. P.), Jr., Effect of surface roughness on eddy current losses at microwave frequencies, J. Appl. Phys., 1949, $20,352$.

[58] Chambers (R. G.), Pippard (A. B.), The effect of preparation on the high frequency surface resistance of metals, Inst. Metals Monog., 1952, 13, 281.

[59] Pacault (A.), Ubersfeld (J.), Les Carbones (1965), Tome I, p. 339.

[60] Pacault (A.), Marchand (A.), Propriétés électroniques des carbones prégraphitiques, J. Chim. Phys., 1960, 57, 873.
[61] InOKuChi (H.), Akamatu (H.), Electrical conductivity of organic semiconductors, Solid State Phys., 1961, 12, 93.

[62] Kronick (P. L.), Labes (M. M.), Organic semiconductors. V. Comparison of measurements on single crystals and compressed molecular complexes, J. Chem. Phys., 1961, 35, 2016.

[63] Mrozowski (S.), Studies of carbon powders under compression Proc. of $3^{\mathrm{e}}$ Conf. Carbon, Buffalo, 1957 , p. 495.

[64] Gutman (F.), Lyons (L. E.), Organic semiconductors, Wiley, 1967.

[65] Eley (D. D.), Organic semiconductors, Research (London), 1959, 12, 293.

[66] Huggins (C. M.), Sharbaugh (A. H.), Dielectric properties of some powdered semiconductors, J. Chem. Phys., 1963, 38, 393.

[67] Snowden (D. P.), Saltsburg (H.), Pereue (J. H.), Jr., The frequency dependent electrical conductivity of powders : nickel oxide, J. Phys. Chem. Solids, 1964, 25, 1099.

[68] Malliaris (A.), Turner (D. T.), Influence of particle size on the electrical resistivity of compacted mixtures of polymeric and metallic powders. J. Appl. Phys., 1971, 42, 614.

[69] Degols (L.), Etude de la relation entre la dispersion électrique et l'adsorption sur poudres semiconductrices et isolantes, J. Chim. Phys., 1969, 66, 1302.

[70] ZeECK (E.), VoITLÄNDER (J.), Untersuchungen an pulferförmingen Halbleitern mit Hilfe von Mikrowellen, Z. Angew. Phys., 1969, 28, 137.

[71] Snowden (D. P.), Saltsburg (H.), Coaxial chamber for measurement of frequency-dependent conductivity in powdered semiconductors, Rev. Sci. Inst., 1963, 34, 1263.

[72] OGawa (T.), Measurement of the electrical conductivity and dielectric constant without contacting electrodes, J. Appl. Phys., 1961, 32, 583.

[73] Henisch (H. K.), ZuCKer (J.), Contactless method for the estimation of resistivity and lifetime of semiconductors, Rev. Sci. Inst., 1956, 27, 409.

[74] Keller (W.), Messung des spezifischen widerstandes von Halbleiterkristallen mit Hochfrequenz, $Z$. Angew. Phys., 1959, 11, 346.

[75] Weingarten (I. R.), Rothberg (M.), Radiofrequency carrier and capacitive coupling procedures for resistivity and lifetime measurements on silicon, J. Electrochem. Soc., 1961, 108, 167.

[76] Miyamoto (N.), Nishizawa (J. I.), Contactless measurement of resistivity of slices of semiconductor materials, Rev. Sci. Inst., 1967, 38, 360.

[77] Kobayashi (H.), Ogawa (S.), Dielectric constant and conductivity measurement of powder samples by the cavity perturbation method, Jap. J. Appl. Phys., 1971, 10, 345.

[78] Abeles (F.), Progress in optics, Vol. 2, Ed. Wolf (North Holland, Amsterdam, 1963).

[79] Proceedings of the symposium on recent development in ellipsometry, Electrical materials laboratory, University of Nebraska, Lincoln, Nebraska, 7-9 août 1968, paru dans Surface Science, 1969, 16. 